\title{
Editorial
}



A través de la historia, científicos de todo el mundo se han dado a la tarea de crear instrumentos tecnológicos que faciliten las labores diarias, sin embargo estas aportaciones creadas para una determinada ciencia como pudiera ser la ingeniería o la astronomía, hoy en día su aplicación se da en el área de la salud; es por ello que mentes brillantes a mediados del siglo pasado crean un mundo alterno, en donde aparatos llamados computadoras, instan y aparecen como máquinas enormes en tamaño y en apariencia, que conforme van creciendo en capacidad, diseño, utilidad y alcance, disminuyen en tamaño hasta crear dispositivos realmente diminutos los cuales se han ido perfeccionando al paso del tiempo en razón de la propia necesidad del ser humano.

Y que al día de hoy sirven por igual al estudiante, a la ama de casa, como al empresario, y es aquí donde el profesional de la salud en especial el profesional enfermero puede hacer uso de esta tecnología en las diferentes funciones, como son la asistencia, pero en dónde lo aplicamos de inicio, es en el cuidado de la persona hospitalizada; por ejemplo al realizar el monitoreo de hemodinámico de una persona en área critica.

En funciones docentes y de enseñanza, al implementar planes de clase a través de las diferentes plataformas; en el aspecto directivo o de gestión mediante la innovación y diseño de formatos digitales que beneficien el registro clínico de las observaciones en el proceso de control y supervisión.

Uno de estos avances sin duda son las aplicaciones que contienen los llamados set o suite, en los que como paquetes alistan programas o aplicaciones de utilidad entre ellos las hojas de cálculo y las bases de datos; De igual forma en la conformación y normativa regulan el manejo y uso del expediente clínico digital.

Y en el área de la investigación al ser un medio generador de información inmediata, tanto de consulta como de distribución de la ciencia

Si bien una de las implicaciones básicas del profesional es contar con la instrucción en el manejo de dichas tecnologías.

En un mundo globalizado y competitivo el obtener datos en forma rápida y precisa nos compete a tomar decisiones en el momento preciso, Sí bien el profesional enfermero tiene como responsabilidad contar con las habilidades y conocimientos tecnológicos para accionar ante un panorama determinado y en donde su habilidad para la toma decisiones será concluyente.

Recordando que como profesionista y profesional asumimos responsabilidades en donde la actualización es inminente, y lo que hace unos minutos era verdad absoluta, hoy en día es historia.

Por lo tanto el estar a la vanguardia en el uso y manejo de la información a través del conocimiento y la tecnología, nos concibe y unifica como gremio a nivel mundial.

Enfocándonos en ello, el contribuir en la historia a partir de la implementación de nuestra revista de enfermería neurológica en un nuevo formato, el digital, en donde al ser un medio de difusión masivo, rebasa las fronteras mismas que la tecnología; coadyuva a alcanzar las metas propuestas. 


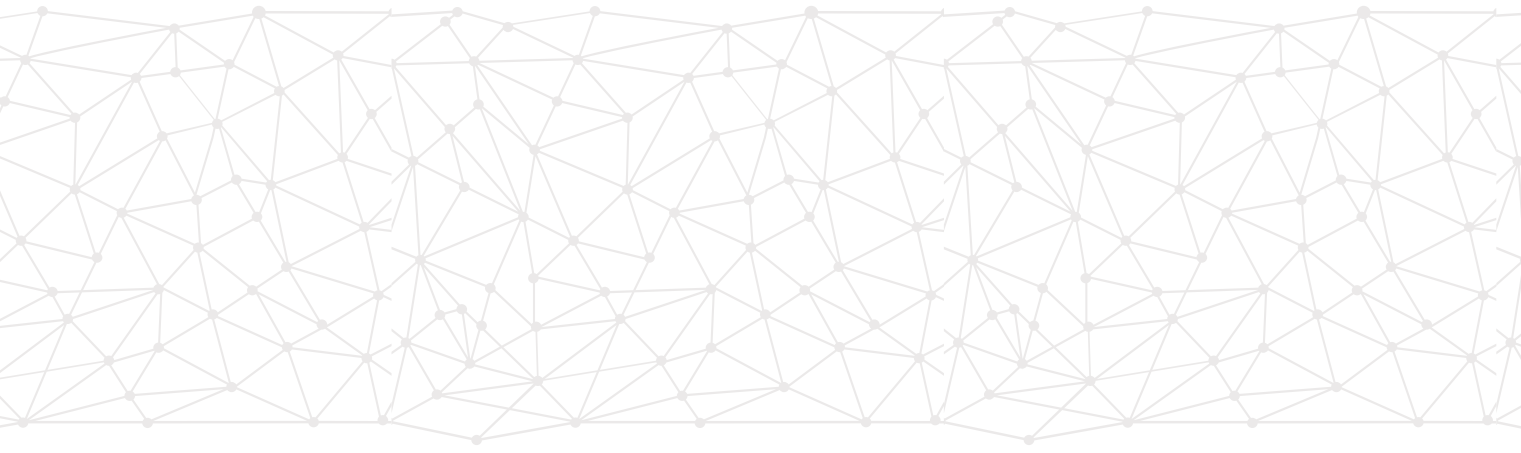

Por ello haciendo rubrica de quien es la Revista de Enfermería Neurológica.

La Revista de Enfermería Neurológica (México) 2015.

Es editada por la Coordinación de Investigación en Enfermería de la Subdirección de Enfermería del Instituto Nacional de Neurología y Neurocirugía Manuel Velasco Suárez. Está dirigida a los profesionales en materia de salud, al ser transdiciplinar por su contenido es consultada por diversos profesionistas en formación especialmente profesionales y estudiantes de enfermería

En ella se presentan artículos científicos que derivan de la investigación relacionada con las neurociencias en enfermería.

Fundamentado en la misión de esta publicación cuya Misión es:

Ser un medio de difusión y comunicación científica en enfermería, distinguido por la generación de nuevos conceptos e ideas, técnicas y procedimientos en el área de las neurociencias, consolidándose como una publicación de vanguardia, al contribuir en la formación y potencialización del capital humano en enfermería, posicionándonos como la revista de mayor calidad, objetividad y prestigio, misma que motiva a la actualización continua en el ámbito disciplinar.

Al ser un medio de difusión científica consideramos

\section{como Visión:}

Instituir el posicionamiento a nivel nacional e internacional la Revista de Enfermería Neurológica, como la principal publicación en su género mediante un plan de mercadotecnia estratégico, de excelencia y eficiencia en el proceso editorial, potencializando nuestro grupo de trabajo, mediante el aprovechamiento óptimo de la infraestructura excediendo así en todo, las expectativas de nuestros suscriptores.

\section{Al tener como Objetivos}

Presentar en forma atractiva y amena temas e innovaciones en el área de las neurociencias, tanto de interés permanente como de actualidad, e informar de los avances más significativos de la ciencia y la tecnología, de manera que los lectores puedan:

- Ser partícipes y beneficiarios de la experiencia del quehacer científico en enfermería.

- Promover y difundir las innovaciones en materia de ciencia tecnológica en enfermería y ciencias de la salud.

- Disponer de información científica dentro de las propias disciplinas de la ciencia y dar elementos que permitan establecer su importancia para la vida social e individual como profesional

- Disfrutar del placer de la creación, difusión y transcendencia del conocimiento.

Al ser un medio de difusión de la ciencia es imperante contar con una plataforma que acerque al profesional en formación ya sea de pregrado o posgrado a información reciente que contribuya en la formación de ese recurso humano y sea potencializado por ello la creación implementación de 


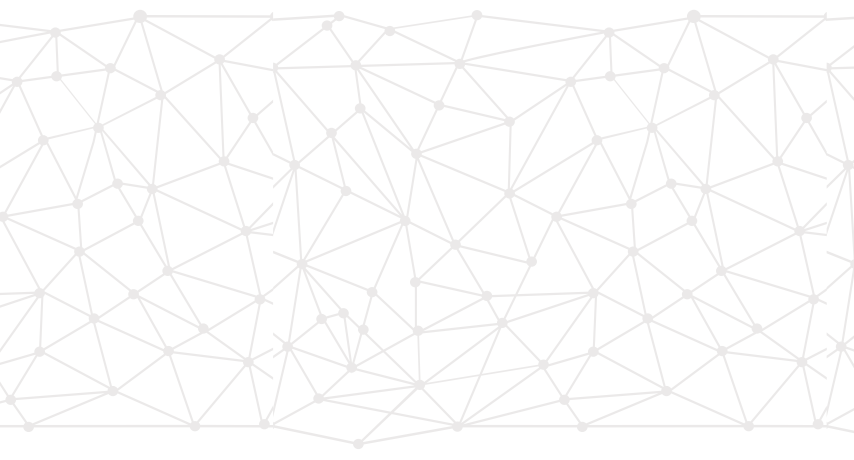

\section{ENFERMERÍA NEUROLÓGICA EN LÍNEA}

La revista está en proceso de creación de la página web para libre acceso a través de la dirección electrónica:

\section{www.revenferneurolenlinea.org.mx}

El proceso editorial de esta en los últimos años se ha fortalecido a partir del equipo editorial el cual está integrado por especialistas en divulgación de la ciencia en medios escritos y digitales, aunado a ello cuentan con una amplia trayectoria editorial aunada a ello la experiencia y expertes profesional en el ámbito de las neurociencias.

Los autores son divulgadores de la ciencia, investigadores, profesores en activo; el proceso editorial inicia al recibir las propuestas de los manuscritos, estos son sometidos a un proceso de revisión y adecuación a fin de mantener y elevar el contenido científico mediante un rigor metodológico cada día más exigente y de esta forma satisfagan el perfil editorial de la revista.

Impacto

Estamos en el proceso de generarlo a razón de la implementación de medios para su registro y análisis.
La revista ha crecido en forma sostenida desde su creación y fortaleciéndose día a día con la colaboración de las personas que han participado en más de una década; misma que se ha convertido en material de consulta frecuente, en las escuelas y facultades de las ciencias de la salud como de enfermería, en virtud de que muchos docentes consideran a la revista como un material de consulta valioso.

La revista se vende por suscripción y se distribuye en eventos académicos como Reuniones de investigación, Jornada de enfermería, Simposio y Encuentros en materia de ciencia en enfermería principalmente.

Por lo tanto como profesional en ciencias de la salud debemos el uso y conocimiento de las tecnologías básico, a la gestión y administración empresarial y al contar con los programas y sistemas de comunicación. Favorecen y facilitan radicalmente su actuar.

Por ello invitamos a que se suscriban y visiten la plataforma, y nos proporciones a través de sus comentarios, ideas que conlleven a mejorar la calidad en los contenidos a partir de sus necesidades como lectores, autores de la ciencia en enfermería

Cordialmente

MCE. Angélica Guadalupe Dávalos Alcázar Editora.

\footnotetext{
10 NWWhw Vol.74. No. 7 enero-abril 2075
} 